$\begin{array}{cccc}\text { S sciendo } & \text { International Conference KNOWLEDGE-BASED ORGANIZATION } \\ \text { Vol. XXV } & \text { No } 2 & 2019\end{array}$

\title{
THE SCIENCE WE NEED IN ORDER TO ACHIEVE PROGRESS IN THE SOCIAL DEVELOPMENT
}

\author{
Venelin TERZIEV \\ “Vasil Levski” National Military University, Veliko Tarnovo, Bulgaria, \\ terziev@skmat.com
}

\begin{abstract}
The aim of this study is to gather sufficient evidence and arguments in order to prove to society that during the process of change in thinking and especially in the effective practical implementation of such educational models, high levels of efficiency can be achieved at all hierarchical levels in key systems for the society and the state.
\end{abstract}

Keywords: science, criterion, publications, impact factor

\section{Introduction}

In the recent years, there has been a lot of debate about measuring science, quality, dimensioning in a way and finding an element for its assessment. Indisputably, this is connected with defining the remuneration of people who have been involved in such an activity. All who deal with the economy of labour and, generally, with human resources management know how difficult and sometimes even impossible it is to give an adequate answer to such a question. The expertise of the personnel who evaluates dimension and even gives an account of this activity should be high enough, and the evaluation just and true enough in order to be accepted by the monitored and evaluated employee. Applying and elaborating various models and technologies in this direction would always bring satisfied and unsatisfied parties. This shouldn't in any way frighten, insult or discourage either party. Achieving maximum justice is a strong enough motive for moving in this very direction of making objective the essential processes that are connected with scientific and research activities. Moreover, life proves that progress is connected with scientific findings.

\section{The science we need in order to progress}

We very often perceive the things rather pragmatically from our own point of view, being satisfied by the explanation that they go well or in an acceptable way at the moment. This acceptable way might be relatively good for a certain moment but by no means is it acceptable and positive for the science process's progress. I repeatedly mention the word "process", because I consider it to have a long duration and its characteristic features, complexity and even contradiction. It should be reviewed, defined and even rationalized by all its participants but mainly by the users. The opposite would be an end in itself in playing science and would bring satisfaction to the person for himself 
without being useful for the others around. You agree that the main benefit in this respect is the applicability of all that scientists and researchers do. Historically, this has been repeated long enough throughout the years, and in not so few cases, the scientific findings have been accepted and evaluated at a much later date in time. The acceptance or denial of a certain scientific activity requires its understanding, perception or approval by the society of the time. This is not always an easy and achievable task for one person or for a group of researchers. Usually, the scientific findings, research papers or works are published in specialized editions that are accessible for a limited circle of people or these editions are not public enough. This further complicates the process of approving or perceiving the circumstances. The established necessity that the scientific works should be published in referred editions, i.e. the ones that contain highly valuable scientific information, has turned them into closed or limited editions where the published information is not accessible. This is also understandable, considering the efforts and work of the relevant teams or of certain researchers willing to get a feedback for their efforts and to be evaluated in a proper and acceptable way.

On the other hand, the scientists' work should reach a wider community because practically it is the potential user of their work. This forces, or the right word is, perhaps, obliges scientists and researchers to present their theses, works and findings at various forums in order to quickly reach a maximum number of users along the various information channels. Explained in this way, it sounds rather simplified, but it is relatively true. One research paper is sometimes presented, shown and debated about at tens of scientific conferences, round tables, symposia and workshops, becoming a complicated, difficult and timeconsuming process. The resistance manifested these on the part of the scientific community in such cases is serious because the search for not traditional approaches in scientific research works breaks up the standard frame of slowness. This "annoys" the scientific community, which is quite introvert and even ossified in its understanding of the popularization of scientific works

My understanding is that almost all variations of a paper presentation are acceptable if they have good results and ensure reliable and authentic feedback. The interrelation between authors and users should be more than warm and open so that the gaps, negative aspects or imperfections could be defined in a sufficiently exact and correct way.

The changes in the Academic Staff Development Act and in the Regulations for its application seek for answers to these questions as they try to establish a system of criteria, which is measurable for the relevant field of higher education and professional direction. This system is serviced by the National Center for information and documents to the Ministry of Education and Science. It is hard to say whether the quantity measures set in this model could ensure the necessary quality coverage. Particularly, if one considers the various evaluation and feasibility systems, as well as whether the different requirements were in operation during various periods. Covering certain up-to-date criteria sometimes leads to the impossibility for some scientists to present adequate information since the older information within the paper appears to be an additional difficulty.

This does not underestimate the efforts that are made in this direction - unified requirements to be introduced and considered at the procedures for habilitation theses for associate professor and professor titles, as well as at the educational and scientific degree "doctor" and the scientific degree "doctor of science".

Naturally, they are differentiated by certain criteria, where the relative weight is put on the number of basic directions: articles and 
reports, published in scientific editions, referred and indexed in worldwide popular data bases with scientific information; articles and reports, published in nonreferred magazines with scientific review or published in edited collective volumes; quotations in monographs and collective volumes with scientific review; quotations or review in non-referred magazines with scientific review; guidance of a successfully graduated Ph.D. candidate; published university textbooks or textbooks that are used in the school networks, etc. Here particular significance is set for the publications that are in the referred systems of Web of Science и Scopus. It is considered that the requirements for such publications are high enough and that the score is high for the published works. This is certainly a matter of perception on the part of the evaluation body for the individual paper itself and for the institution as a whole.

All this also directly corresponds to various rating systems that consider particular articles in particular editions and classify the institutions after these indicators, including reviews of these scientific reports, articles or announcements. This seems quite acceptable and adequate as an evaluation technology, but the result does not always correspond to the reality. Such an evaluation is also made in Bulgaria and at the end of each year the results from the previous one are presented. The time for evaluation is not accidentally chosen. Indexing of the relevant publications in both major systems is a long lasting period, and in some cases it continues over a year. Sometimes, in these cases, there is the possibility for part of the publishing activity of the scientists to be incorrectly and not accurately reported. In some of the higher education institutions, progress is due to one or several scientists, who have formed a team and have established themselves in the academic community, being able to freely publish in this type of editions. This somehow distorts the presented information and leads to quantity accumulations that do not correspond to the institution's overall evaluation. In some cases, if we exclude these few scientists from the structure of the relevant higher education institutions, the resulting data would be insignificant.

Another evaluating indicator is the time the particular academic position is held. If it is acceptable for the people who are employed only on this basis, then it is unrealistic and even unachievable for the others. It could not be objective enough at least because of the fact that the particular skills, knowledge and competencies do not always depend on the time period.

"Detaching" the scientific research works from the social development is also a major, not insignificant problem in its essence. Here the questions are related to their usefulness, as well as to when and how. One has to exclude the completely theoretical works that have other meaning and necessity.

The opinion of Celia Luterbacher from Switzerland is very interesting. She has interesting interpretations in this direction. She asserts that one ruling ruthless principle within the scientific community is "I publish, so I exist”. In this respect, special meaning is given to the quantity measures, which consider the number of publications and the referrals to them. In Switzerland, a reform that is connected with change in this direction is under way. In recent years, quantity indicators are more and more often used for the sake of the quality ones and practically there is no way to motivate scientists, not to mention that this process could reduce the quality of scientific research. All this would be connected with a change in the national strategy, which should consider the various disciplinary and institutional requirements, while applying differentiated evaluating practices.

Introducing such evaluating systems would also raise a number of questions and critical notes. The first one among them is who and in what way this quality evaluation would be achieved. So far, the adopted technology 
is the $\mathrm{h}$ index, which considers the relevant number referrals to a certain article and the other time indicator, which is used for the first one to be defined for the last 5 years. The various systems would show various figure evaluations because the information data bases are different in terms of completeness and accessibility. Different assessments could be used for the various fields of higher education, which again would give relatively true and exact information. Some would say that it is better to have a kind of system rather than no system at all, and they would be absolutely right. The lack of a system of measurable criteria led to a number of paradoxical differences in the levels of evaluation. I could not say whether this defines the quality of the candidate for holding a certain academic position, but it is a peculiar testimonial for the expertise of the corresponding commission. In this case, this is a scientific jury who has to evaluate and rank the candidates. Usually, the competitions in our country are held with only one candidate. For some professional fields, it is just the lack of other candidates, but sometimes it is also the impossibility to occupy the relevant position because of a number of objective circumstances. These are connected with the difficulties in the development of the academic structure in the recent decades, the unappealing nature of the field or lack of financing for scientific research. Sometimes the locality of the relevant higher education institution is not acceptable for the candidates, too. In other cases, only candidates from the interior environment are tolerated despite of the fact that the possible number from the exterior environment would be big enough. In this case, the experts' work would be much more difficult and the competition greater. The possible choice would fall on more than one candidate. Another issue of correctness is the following choice from the scientific and faculty council. In many cases, they are quite heterogeneous in terms of fields and interests, appear to be difficult and are not always connected with objective evaluation and, in the long run, with correct choice. In this case, the question is whether these are competent enough to change the end result with their choice, despite the fact that this is integrated in their legal powers. And if we leave this aside as a secondary problem, the searching and finding of appropriate candidates on this quite limited labor market of scientists and researchers leads to a closed cycle. It is not a secret that part of the universities is place of subsistence for whole families for years already. And there is nothing bad in it because a profession is handed down from one generation to another, but to what an extent the abilities of our children tally with our life ones and in particular, with our scientific interests. It is hard this way, but it is an easy enough option, supported by the academic autonomy. Such a study would give frightening results of closeness and interrelations. Quite interesting developments are observed in the smaller communities, where the processes are selfregulated by this factor.

The change we advocate looks for certain justice and accuracy, but would hardly achieve it only by these corrections in the legislation. It should rather be connected with several corrections in the management of the system. Mandates, creativity, strategies and etc., are very often spoken about in the most common case, connected with modeling the adjustments of the general assembly in a certain way, which sometimes is on the verge of the norms and the law. Despite that, the procedures are legal and not very moral. There are constantly many proofs in this direction.

Not always the democratic rules choose the most skillful and the most competent, and sometimes they even do not give a part of the scientists the opportunity for contribution because of a number of reasons.

This is a common problem, which is applicable with greater power for the academic community. Here individualities 
are many and in various directions. Sometimes the uniting is for the sake of a choice, which is rather connected with other circumstances, not with the individual's qualities. The change in this direction is possible if, at this choice, there is a contest based on maximum objective criteria. [1], [2]

\section{Conclusions}

My personal sense of satisfaction is rooted in the belief that the work of a scientist has a certain extent of usefulness and significance and puts Bulgaria in a visible position in the scientific societies in which we work. For me it is already clear that enmity, malice and even envy are characteristics that accompany the hard way. I put it on the national peculiarities or on the changes from one generation to the other. Anyway, we should not forget that the efforts should be constant and achieving progress in a scientist's development should be looked for.

\section{References}

[1] https://www.lex.bg/laws/ldoc/2135696665.

[2] https://lex.bg/en/laws/ldoc/2135680028. 